\title{
Abzymes in the Diagnosis of Bronchial Asthma
}

\author{
Cyrille Alode VODOUNON* \\ Laboratoire de Biochimie et de Microbiologie, Ecole Normale Supérieure de Natitingou, University of Parakou \\ *Corresponding author: sweetiebj@yahoo.fr
}

\begin{abstract}
The objective of the present work was to study the predominance of abzymes in the diagnosis of Bronchial Asthma. The study was carried out using the blood serum of several groups of patients, including patients with Bronchial Asthma ( $\mathrm{n}=15)$, allergic Rhino-conjunctivitis (polypus)(n=15), Systemic Lupus Erythématosis( $\mathrm{n}=10)$ and Auto-Immune Thyroiditis $(\mathrm{n}=10)$. Detection of IgG auto-AB against DNA was carried out by Enzyme-linked immunosorbent assay (ELISA) and the catalytic activity of antibody against DNA was determined by electrophoresis method. The results of our study revealed abundant auto-antibodies to DNA in the blood vessels of the patients with bronchial asthma and there was a direct correlation dependence $(r=0.0005)$ between the level of auto-antibodies to DNA and the severity of the disease. Circulating auto-antibodies in blood serum possess catalytic activity of DNA which is associated with the degree of severity of asthma. Thus, autoantibody can be used as serologic markers for the diagnosis of BA and can even be suitable, according to their concentration, for determining the degree of severity of the disease.
\end{abstract}

Keywords: Abzyme, autoantibody, Bronchial Asthma

Cite This Article: Cyrille Alode VODOUNON, “Abzymes in the Diagnosis of Bronchial Asthma.” American Journal of Biomedical Research, vol. 5, no. 2 (2017): 35-38. doi: 10.12691/ajbr-5-2-3.

\section{Introduction}

Rottem [1] reported that the Allergy and autoimmunity can have common mechanisms in the pathogenic pathways. According to some authors, asthma could be intrinsic causes as in autoimmune reaction or a genetic predisposition, although there is no study that has confirmed this. The analysis of auto-antibody profiles has shown to be important in the diagnosis of some auto-immune diseases, such as anti-dsDNA and anti-Sm auto-antibodies in the systemic lupus erythematosus (SLE) [2]. According to Beland, [3] Vitozzi, [4] Eirini, et al [5], anti-LC1 (liver cytosol type 1 antibody) and anti-SLA (antibody against soluble liver antigen) auto-antibodies can be regarded as a serological markers of autoimmunity and need to be tested while investigating autoimmunity. Abzymes (derived from antibody and enzymes) is a monoclonal antibody with catalytic activity. They are usually artificial constructs, but are also found in normal humans and patients with autoimmune diseases. Abzyme's appearance is the general tendency of the answer to the immune system in the severe contaminations. We deduce that catalytic activity can find its reflection in the natural potential defence of immune system for different types of autoimmune disturbances. Therefore, the objective of this study was to study the predominance of abzymes in the diagnosis of Bronchial Asthma (BA).

\section{Materials and Methods}

The study was carried out using the blood serum of several groups of patients, including patients with Bronchial Asthma $(\mathrm{n}=15)$, allergic Rhino-conjunctivitis (polypus) $(\mathrm{n}=15)$, Systemic Lupus Erythématosis $(\mathrm{n}=10)$, and Auto-Immune Thyroiditis) $(\mathrm{n}=10)$ (Global Strategy of treatments and prophylactics of the bronchial asthma, 2007). All the patients were apprised and they agreed through written consent provided. The severity of asthma in these patients was assessed according to the Global Initiation for Asthma guideline [6,7]. The diagnosis of BA was established on the basis of data of allergic anamnesis. The work was performed in accordance with the rules of the Ethics Committee in the laboratory of Clinical Immunology and Allergy of RKB and with the regulations of the Ministry of Health of the Russian Federation in compliance with the Helsinki declaration. Detection of IgG auto-AB against DNA was carried out by Enzyme-linked immunosorbent assay (ELISA) [8]. For the study of the catalytic activity of IgG auto-antibodies against DNA in the blood serum the reaction was conducted in vitro. Commercial preparation of Plasmids DNA ${ }_{\mathrm{p}} \mathrm{BR} 322$ extracted from E.coli XL-1Blue («Sib Enzyme, Russia) was used as substratum. The catalytic activity of antibody against DNA was estimated by the transformation of the supercoiled plasmid DNA pBR322 in the circular and linear form by electrophoresis. Electrophoresis was performed in $0.7 \%$ of agarose gel, prepared on TBE containing $0.09 \mathrm{M}$ Tris-OH, 0.09M boric acid, 0.002M EDTA and at $\mathrm{pH} 8.0$ 8.2 with a voltage of $3-5 \mathrm{~V} / \mathrm{cm}$ for 1 hour $30 \mathrm{~min}$ at $20{ }^{\circ} \mathrm{C}$. The gel stained for $15 \mathrm{~min}$ in the TBE containing $1 \mu \mathrm{g} / \mathrm{ml}$ ethidium bromide. The evaluation of the results of the plasmid DNA pBR322 was carried out on gel analyzer system of brand Chemi Doc TMX RS (Bio-Red Laboratories, USA). 


\section{Results and Discussion}

To evaluate the possible involvement of the autoimmunity in the pathogenesis of bronchial asthma, the availability and the level of circulating IgG antibodies (auto-antibodies) were determined in the blood serum of patients' peripheral blood and relatively healthy donors. According to the results of ELISA test, some IgG antibodies to ${ }_{\mathrm{N}} \mathrm{DNA}$ were found in patients with atopic (BA) and non-Atopic Bronchial Asthma. As shown in Figure 1, in patients with asthma regardless of the pathogenesis of the disease, it was revealed an increase in the level of IgG anti- ${ }_{N}$ DNA compared to the control group. In the patients with infectious dependent variant the level reached was significant $(r<0.5)$.

Given the particularities of the occurrence of circulating auto antibodies to DNA in patients with atopic dermatitis and infectious-dependent variant of bronchial asthma, we have increased samples of patients with atopic bronchial asthma and investigated on the character of distribution of the levels of anti-DNA IgG in blood with the progression in ABA, i.e., in the light, average and high severity of the disease. Figure 2 shows that there was a significant tendency $(r<0.01)$ of growth in AAT to NDNA to the extent of the deterioration of the disease. Correlation analysis showed a direct correlation between the level of ${ }_{\mathrm{N}} \mathrm{DNA}$ and the severity of bronchial asthma (Figure 2). Thus, the main contribution to the emergence of auto antibodies to ${ }_{N} \mathrm{DNA}$ in patients with $\mathrm{ABA}$ makes the severity of the disease.

Therefore, auto antibodies found in the peripheral blood of patients with asthma were studied for the presence of catalytic activity. To establish the function of the enzyme system, the serum of Systemic Lupus Erythematosus (SLE) and Auto-Immune Thyroiditis (AIT) were used (Figure 3), and which were characterized by the presence of antibodies abzymes with the catalytic activity of DNA. DNA activities of antibodies serum of patients with asthma were evaluated based on their ability to turn substrate-superspiral DNA pBR322 into the ring and linear form.

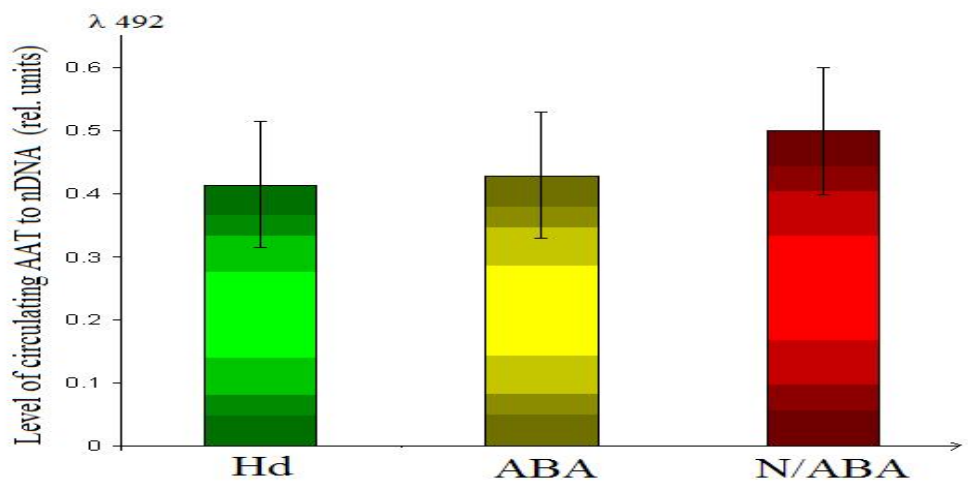

Figure 1. Dependence level of antibodies to ${ }_{N} D N A$ bronchial asthma variant: BA- Bronchial Asthma, N/ABA - Non Atopic Bronchial Asthma; Hdhealthy donor

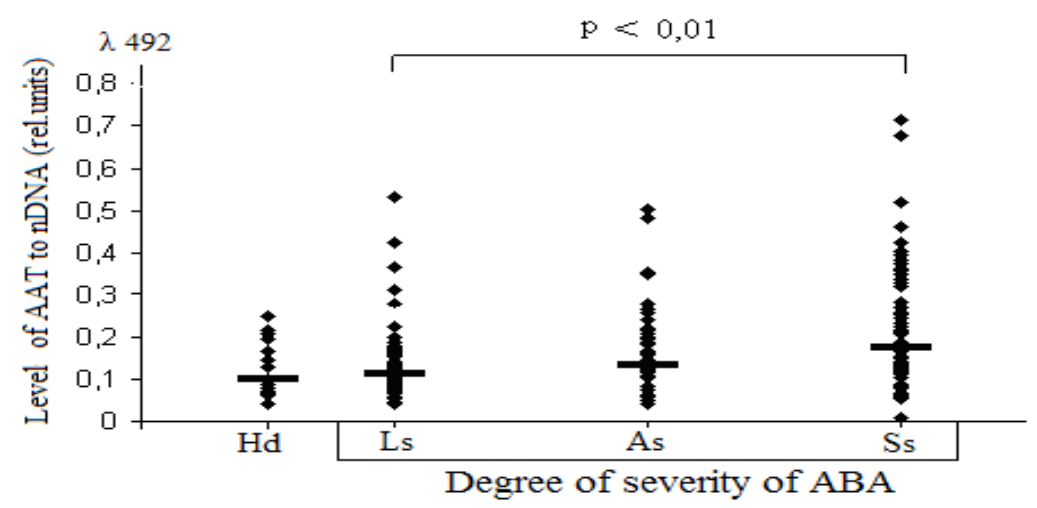

Figure 2. The distribution of the levels of circulating IgG auto antibodies to DNA in the surveyed contingent based on the severity of BA

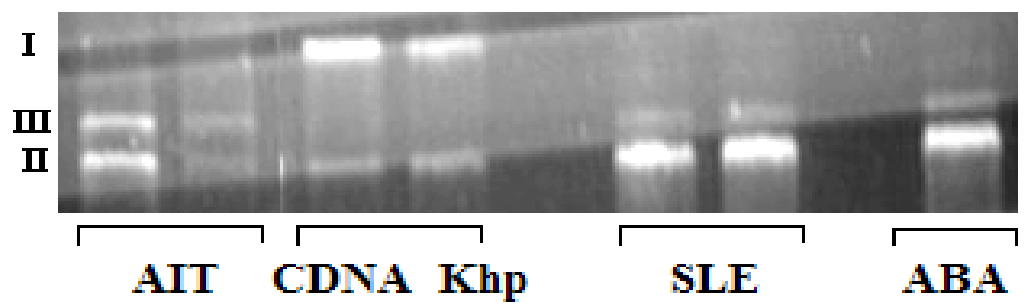

Figure 3. Expression of DNA activity of circulating AAT to nDNA in serum of patients with systemic lupus erythematosus (SLE) and a light form of Auto-Immune Thyroiditis (AIT) and with Bronchial Asthma. Systemic Lupus Erythematosus (SLE), a light form of Auto-Immune Thyroiditis (AIT) based on the hydrolysis of plasmid DNA pBR 322. Reaction is after 24h. I -super spiral, II-ring, III-linear - structural forms of pBR322; CDNA - DNA without reactionary environment, $\mathrm{K}_{\mathrm{hp}}$ - DNA+ serum of healthy people 


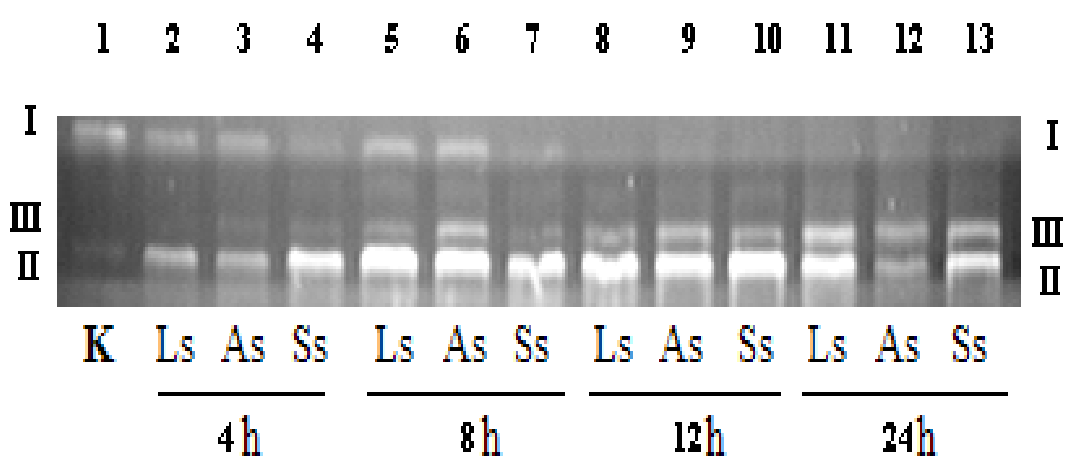

Figure 4. Alteration in the activity at the ${ }_{\mathrm{N}} \mathrm{DNA}$ serum of patients with asthma based on incubation time $\left(37^{\circ}, 24\right.$ hours) and the severity of the disease. I-super spiral, II-ring, III-linear - structural forms of pBR322; CDNA - DNA without reactionary environment; LMS - light, medium and severe forms of asthma, respectively

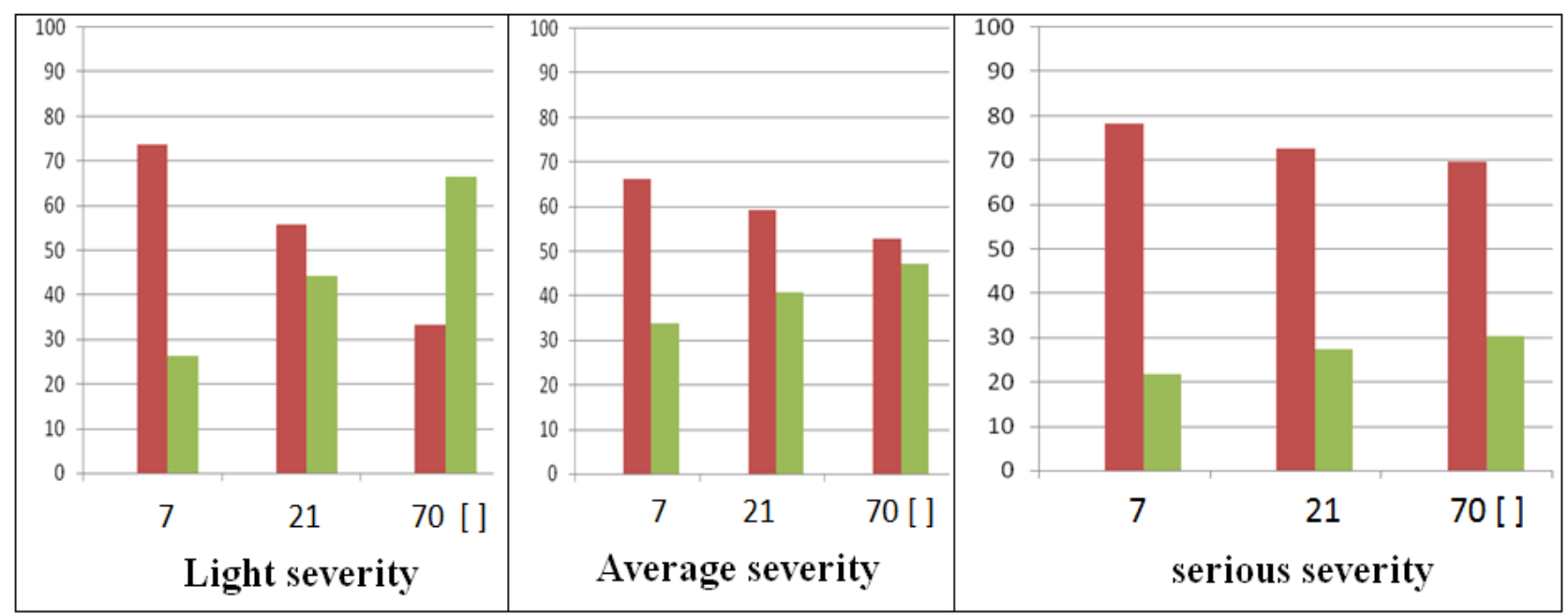

Figure 5. The study of substrate specificity of circulating antibodies serum of patients with Bronchial Asthma based on the severity of the disease. Quantity of introduced protein per sample: 7. 21. $70 \mu \mathrm{g} / \mu \mathrm{l}$. Time of incubation $\left(37^{\circ}, 12\right.$ hours). Hydrolysis product pBR322 (form DNA) in \%. Red color: II (circular), Green color: III (lineair)

Figure 3 illustrates that the serum of patients with BA possesses catalytic activity of DNA with the serum of patients with autoimmune thyroiditis. Then, the DNA catalytic activity level of antibodies' serum of patients with asthma according to the severity of the asthma (within $24 \mathrm{~h}$ ) was studied.

Figure 4 shows that the dynamics in the growth of the number of hydrolysis products were identified based on the time. The level of catalytic activity of DNA depends on the severity of asthma: auto-antibodies serum of patients with severe asthma were more active and show the affinity to monofilament areas of super spiral DNA (form I), as proved by the increase in the circular DNA (form II) (Figure 4, lane 4). After $12 \mathrm{~h}$, the reaction of the whole super spiral DNA entered the ring shape, thus, the influence of protein serum on the substrate specificity of catalytic activity of antibody against DNA according to the severity of the disease was studied (Figure 5). The results showed that the substrate specificity of antibodies was correlated with the severity of the disease. According to Figure 5, the nature of the accumulation of the products from hydrolysis of plasmid DNA pBR322 of circulating antibodies, patients with a severe form of BA possessed endonucleases activity and have a preference to a chain of DNA- after 12 hours in the reaction mixture among the products of hydrolysis and approximately $78 \%$ of circular DNA were found. Increasing 10 times the concentration of protein changed slightly the number of circular DNA (up to 69.5 percent).

The fact is that in solutions with a small ionic force $(0.15 \mathrm{M} \mathrm{NaCl})$ and the neutral or light basic solution, plasmid DNA pBR322 had "super-twisted" structure as a result formed over spiral (form I). The formation of supertwisted forms of DNA was followed by the denaturing of a certain area of molecules which disappeared while changing the DNA from form I to form II, i.e. as a result of a single gap which was the instant recovery of hydrogen bonds in denatured division of the molecule. These properties of DNA pBR322 make them easy targets for the study of endo-nucleases activity of enzymes and antibodies-abzymes (thanks to the attribute of DNA pBR322, it was really easy to target them for the study of endo-nucleases activity of enzymes and antibodiesabzymes).

Therefore, based on the stability of the bi-filament circular DNA pBR322 to the action of the studied circulating antibodies of patients with severe form of BA revealed that the addition of excessive amounts of antibodies of patients with severe asthma or the rise in the reaction time did not lead to a complete transfer of circular DNA (form II) into the linear (form III). The circulating auto-antibodies in blood serum of patients with light asthma were heterogeneous. The analysis of results of Figure 5 shows that in the serum of patients with the light 
form of ABA along with antibodies to monofilament substrate, the circulating antibodies were specific to bifilament DNA, because there was a dynamics of hydrolysis of circular DNA and which increases the number of linear DNA (form III) (up to 68.5) at the rising level of protein serum in the reactionary environment. These data could serve as control and the lower catalytic activity of DNA of antibodies, abzymes, and specific to double-stranded DNA. Thus, in the blood serum of patients with BA we have detected antibodies to catalytic activity of DNA - abzymes, i.e. expressions of autoimmune disease development.

Tedeschi and Asero [9] established that the finding of antinuclear antibodies and antibodies against antigens of the epithelial bronchi cells in patients with non-allergic asthma involves autoimmune basis of the disease, and they further reported the involvement of auto-regency mechanism for allergic (atopic) asthma. Thus, the search for new biomarkers related to the severity of asthma could be very promising. It should be noted that the question of antinuclear in the blood of patients with asthma were studied long time ago, but the results were contradictory. Even in the studies of Howard and Ellsworth (1965), it was demonstrated that the method of anti-nuclear in the lungs epithelium cells was not available. According to Lidor et al. [10] individual with anti-nuclear antibodies was $21 \%$ among patients with BA against 55\% with non atopic BA, 25\% among patients with bronchitis, and 16\% of relatively healthy persons. The results of our works illustrated that the presence of circulating AAT to ${ }_{\mathrm{N}} \mathrm{DNA}$ in the blood serum of adults with BA and demonstrated through mathematical analysis methods, that the direct correlation was most likely established between the level of the DNA and the severity of Asthma $(r=0.0005)$, as it is also known that the IgG has the ability to be recorded in the tissues, it could be assumed that this is one of the damage mechanisms of the tissue-target during a progressive course of the disease and the development of autoimmune form of BA. Circulating auto-antibodies in blood serum have catalytic activity of DNA. In blood serum of patients with the light form of BA along with antibodies specific to monofilament substrate, there are circulating antibodies specific for hydrolyzing bi-filament DNA. In the serum of patients with severe asthma were mostly antibodies to monofilament DNA. Thus, in the blood serum of patients with BA was found antibodies with different substrate specificity and a different level of catalytic activity of DNA - Abzymes. It was suggested that these "abzymes" may be directly involved in the removal of debris produced by the metabolism of organism under physiological conditions [11]. The presence in normal human milk of catalytic antibodies with a protein kinase activity and antibody with DNase activity suggests a protective role of "abzymes" under physiological conditions [12]. Nevertheless, most studies on human being showed that the prevalence of "abzymes" increases in pathological conditions, especially during autoimmune diseases [11]. Our results are consistence with those of their works which described asthma by characterizing the relationship of allergic diseases and autoimmunity as a paradigm of autoimmune diseases [13].

\section{Conclusion}

When taking into consideration all these facts, we can deduce that the degree of bronchial asthma severity reflects the transition of the disease to autoimmune variant of bronchial asthma, which is considered to develop in the further progression and the intensification of the course of atopic and non atopic bronchial asthma. And the discovery of antibodies in the serum of peripheral blood in asthma and analysis of the relative DNA activity of auto antibodies may serve as an additional criterion for the diagnosis of asthma even in the early stages and evaluation of the effectiveness of the treatment.

\section{References}

[1] Rottem M, Shoenfeld Y. Asthma as a paradigm for autoimmune disease. Int. Arch. Allergy. Immunol 2003, 3: 210-214.

[2] R Brouwer, G J D Hengstman, W Vree Egberts, H Ehrfeld, B Bozic, A Ghirardello, G Grøndal, M Hietarinta, D Isenberg, Autoantibody profiles in the sera of European patients with myositis. Ann Rheum Dis 2001; 60: 116-123.

[3] Beland K, Lapierre P, Marceau G, Alvarez F: Anti-LC1 autoantibodies in patients with chronic hepatitis $\mathrm{C}$ virus infection. J Autoimmun 2004, 22:159-166.

[4] Vitozzi S, Lapierre P, Djilali-Saiah I, Marceau G, Beland K, Alvarez F: Anti-soluble liver antigen (SLA) antibodies in chronic HCV infection. Autoimmunity 2004, 37:217-222.

[5] Eirini I Rigopoulou, Maria Mytilinaiou, Ourania Romanidou, Christos Liaskos and George N Dalekos Autoimmune hepatitisspecific antibodies against soluble liver antigen and liver cytosol type 1 in patients with chronic viral hepatitis. Journal of Autoimmune Diseases 2007, 4:2.

[6] National Heart, Blood, and lung Institute, Global strategy for asthma management and prevention: WHO|NHLBI workshop report, M.D.Bethesda, National Heart, Lung, and Blood Institute; publication ${ }^{1}$ 1995, 95-3659.

[7] National Asthma Education and Prevention Pro-gram, Expert panel report 2: guidelines for the management of asthma, M.D.Bethesda, National Institutes of Health, Publication ${ }^{1}$. 1997, 97-4051.

[8] Goldsby R.A, Kindt T.J, Osborn B.A. Enzyme-linked Immunosorbent Assay Immunology 2003, 5: 148-150.

[9] Tedeschi A, Asero R. Asthma and autoimmunity: a complex but intriguing relation. Expert Rev Clin Immunol 2008; 4(6): 767-776.

[10] Lidor, Y, Topilsky M, Spitzer S.A. Yeehoshua H. Autoimmune antibodies in intrinsic (non-atopic) asthma. Ann. Allergy 1980; 5: 296-298.

[11] Sébastien Lacroix-Desmazes, Jagadeesh Bayry, Michel D. Kazatchkine et Srini V. Kaveri. Anticorps catalytiques ou abzymes; Catalytic activity of antibodies. médecine sciences 2003; 5: 519-522.

[12] Sébastien Lacroix-Desmazes, Alexandre Moreau, Jagadeesh Bayry, Michel D. Kazatchkine, Srinivas V. Kaveri Activité hydrolytique des anticorps anti-F VIII inhibiteurs chez les patients hémophiles A. Hematologie 2002; 6: 422-426.

[13] Tirosh, A, Mandel D, Mimouni F.D et al. Autoimmune diseases in asthma. Ann. Intern. Med 2006; 12: 877-883. 